\title{
Correction \\ Correction: Raasakka, A.; Kursula, P. Flexible Players within the Sheaths: The Intrinsically Disordered Proteins of Myelin in Health and Disease. Cells 2020, 9, 470
}

\author{
Arne Raasakka ${ }^{1}$ (D) and Petri Kursula ${ }^{1,2, *(\mathbb{D})}$ \\ 1 The Department of Biomedicine, University of Bergen, Jonas Lies vei 91, NO-5009 Bergen, Norway; \\ arne.raasakka@uib.no \\ 2 Faculty of Biochemistry and Molecular Medicine \& Biocenter Oulu, University of Oulu, Aapistie 7A, \\ FI-90220 Oulu, Finland \\ * Correspondence: petri.kursula@uib.no; Tel.: +47-55-586-438
}

check for updates

Citation: Raasakka, A.; Kursula, P. Correction: Raasakka, A.; Kursula, P. Flexible Players within the Sheaths: The Intrinsically Disordered Proteins of Myelin in Health and Disease. Cells 2020, 9, 470. Cells 2022, 11, 662 https://doi.org/10.3390/ cells11040662

Received: 9 July 2021

Accepted: 14 July 2021

Published: 14 February 2022

Publisher's Note: MDPI stays neutral with regard to jurisdictional claims in published maps and institutional affiliations.

Copyright: (c) 2022 by the authors. Licensee MDPI, Basel, Switzerland. This article is an open access article distributed under the terms and conditions of the Creative Commons Attribution (CC BY) license (https:// creativecommons.org/licenses/by/ $4.0 /)$.
The authors would like to make corrections to their review [1] in the light of two recently retracted papers [2,3]. These papers (citations 202 and 203 in the original publication) were cited and the information within was discussed in our review, as well as used in the preparation of Figure 8. We have now removed these citations and all information from the two retracted papers by modifying our review text, as described below.

The following text from Section 2.7 has been updated by replacing citation 202 with citation 200: "The interaction is thought to be mainly mediated by the DRP2 spectrin repeat domain, with possible involvement of the adjacent WW domain and NLS2/NLS3 in L-PRX (Figure 8b) [13,202]".

The following text from Section 2.7 has been deleted: "The NLS2/NLS3 region mediates a self-interaction within L-PRX by binding to the L-PRX acidic $C$ terminus, although it is unclear whether the interaction is intramolecular or if two L-PRX molecules may interact [203]".

The following text from Section 2.7 has been deleted: "The C-terminal acidic region is rich in Glu, which is believed to be the basis of the interaction with NLS2 and NLS3 of the basic region (Figure $8 b$ ). The collective negative charge is likely to play a profound role in the association, although one specific point mutation, E1259K, had a significant inhibitory effect [203]".

The following text from the end of paragraph eight in Section 2.7 has been deleted: "(see below)".

The following text from Section 2.7 has been deleted: "Finally, the self-association of the acidic domain with the tripartite NLS might harbor similar roles in either nuclear export or inhibition of DRP2 and/or integrin $\beta 4$ binding [203]. It remains to be determined, whether the mechanism involves two interacting L-PRX molecules or an intramolecular switch within a single L-PRX molecule that concurrently forms hetero- and homodimers via the PDZ domain".

The following paragraph in Section 2.7 has been deleted: "Bringing several L-PRX molecules together through the aforementioned interactions could result in large-scale assemblies that undergo liquid-liquid phase separation. This could harbor a regulatory role in the formation and stability of the periaxinosome, for instance through the controlled release of free L-PRX to the cytosol or to the membrane surface [216]. The binding of ezrin to L-PRX, or S-PRX heterodimerization with L-PRX, might function as a control mechanism for the formation of phase-separated compartments in the periaxinosome".

The following text from Section 3.2 has been deleted: "The only missense mutation that has a known effect is E1259K, which abolishes the internal L-PRX interaction [203]".

The following text from Section 4 "The intramolecular interaction within L-PRX and the potential regulatory role of S- and L-PRX heterodimerization need to be characterized at 
the molecular level, in order to shed light on PRX nuclear trafficking and the formation of the periaxinosome." has been modified to: "The potential regulatory role of S- and L-PRX heterodimerization needs to be characterized at the molecular level, in order to shed light on PRX nuclear trafficking and the formation of the periaxinosome".

Figure 8 has been modified. The updated figure and figure legend are shown below.

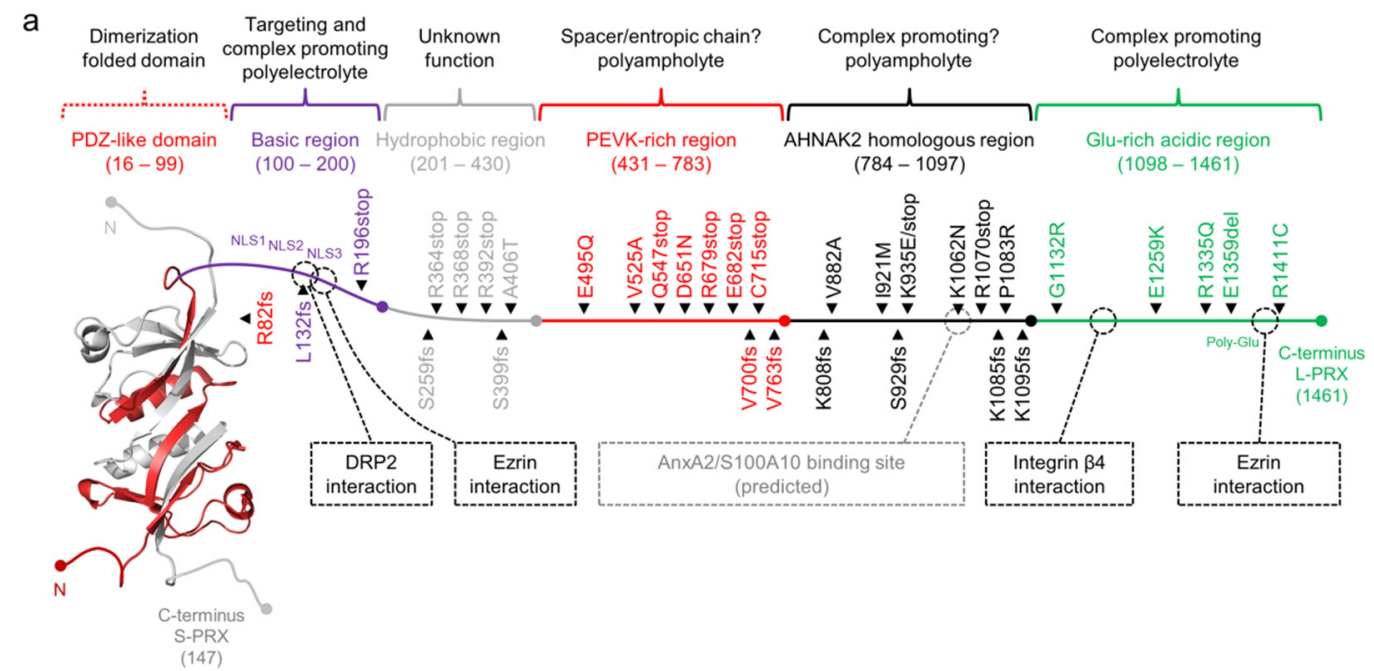

b

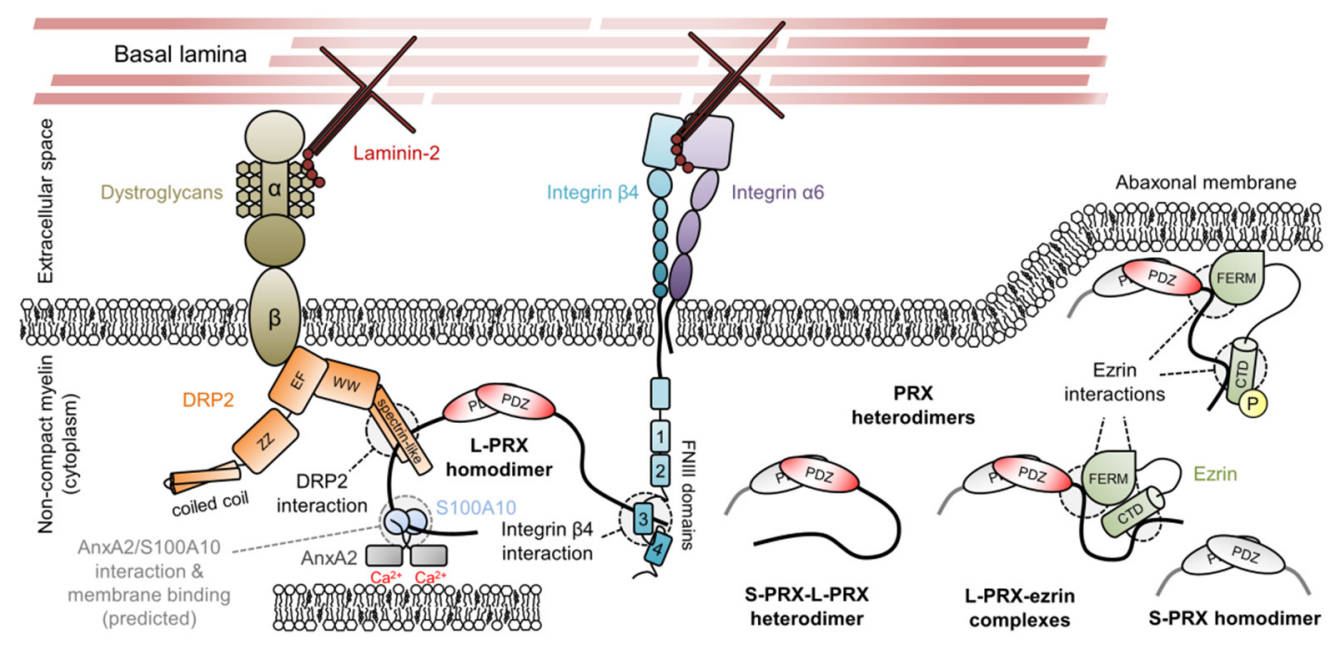

Figure 8. The structure and interactions of periaxin. (a) A schematic of a PRX heterodimer, with S-PRX in gray and L-PRX colored based on region, with the PDZ domain in red. L-PRX, apart from the PDZ-like domain, is predicted to be disordered [45], and can be divided into separate regions based on sequence composition. Peripheral neuropathy mutations are indicated alongside L-PRX. Dashed boxes and lines denote protein-protein interactions. L-PRX contains a predicted AnxA2 and S100A10 binding region, as reported earlier for AHNAK $[195,196]$. See Table 1 for mutation details. (b) L-PRX is an assembler within abaxonal non-compact myelin, linking dystroglycans and integrins together in membrane appositions, forming the periaxinosome. These interaction partners connect the Schwann cell basal lamina to the Schwann cell cytoplasm. S-PRX forms heterodimers with L-PRX, which might allow regulation of the cytoplasmic assembly as well as the nuclear export of L-PRX. Ezrin in complex with hetero- or homodimeric L-PRX might have relevance in such regulations, especially considering its phosphoregulated membrane-binding activity [197]. The function of the S-PRX homodimer is unknown. The significance of the putative L-PRX/AnxA2/S100A10 ternary complex could involve linking the entire assembly via AnxA2 and $\mathrm{Ca}^{2+}$ to the underlying membrane, possibly forming a structural basis for membrane appositions that line Cajal bands in myelinating Schwann cells. 
Table 1 has been modified. The updated table is shown below.

Table 1. PRX mutations, related neuropathies and potential molecular mechanisms.

\begin{tabular}{|c|c|c|c|}
\hline Mutation $^{1}$ & Neuropathy & (Potential) Molecular Impact & Reference(s) \\
\hline R82fs & DSS & Tail loss; loss of interactions & [258] \\
\hline L132fs & CMT4F & Tail loss; loss of interactions & [259] \\
\hline R196stop & CMT4F & & [260] \\
\hline S259fs & CMT4F & $\begin{array}{c}\text { Loss of hydrophobic, PEVK-rich, AHNAK2 } \\
\text { homology and acidic regions; loss of interactions }\end{array}$ & [261] \\
\hline R364stop & CMT4F & $\begin{array}{c}\text { Loss of PEVK-rich, AHNAK2 homology and acidic } \\
\text { regions; loss of interactions }\end{array}$ & [262] \\
\hline R368stop & DSS & $\begin{array}{c}\text { Loss of PEVK-rich, AHNAK2 homology and acidic } \\
\text { regions; loss of interactions }\end{array}$ & [263] \\
\hline R392stop & DSS & $\begin{array}{c}\text { Loss of PEVK-rich, AHNAK2 homology and acidic } \\
\text { regions; loss of interactions }\end{array}$ & [264] \\
\hline S399fs & CMT4F & $\begin{array}{c}\text { Loss of PEVK-rich, AHNAK2 homology and acidic } \\
\text { regions; loss of interactions }\end{array}$ & [265] \\
\hline A406T & DSS & & [263] \\
\hline E495Q & DSS & & [263] \\
\hline V525A & $\mathrm{CMT} 4 \mathrm{~F}$ & & {$[260,266]$} \\
\hline Q547stop & CMT4F & $\begin{array}{l}\text { Loss of PEVK-rich (partial), AHNAK2 homology } \\
\text { and acidic regions; loss of interactions }\end{array}$ & [261] \\
\hline D651N & CMT4F & & [267] \\
\hline R679stop & DSS & $\begin{array}{l}\text { Loss of PEVK-rich (partial), AHNAK2 homology } \\
\text { and acidic regions; loss of interactions }\end{array}$ & [264] \\
\hline E682stop & CMT4F & $\begin{array}{l}\text { Loss of PEVK-rich (partial), AHNAK2 homology } \\
\text { and acidic regions; loss of interactions }\end{array}$ & [261] \\
\hline A700fs & CMT4F & & [268] \\
\hline C715stop & DSS & $\begin{array}{l}\text { Loss of PEVK-rich (partial), AHNAK2 homology } \\
\text { and acidic regions; loss of interactions }\end{array}$ & [258] \\
\hline V763fs & DSS & $\begin{array}{l}\text { Loss of PEVK-rich (partial), AHNAK2 homology } \\
\text { and acidic regions; loss of interactions }\end{array}$ & [263] \\
\hline K808fs & $\mathrm{CMT} 4 \mathrm{~F}$ & $\begin{array}{l}\text { Loss of AHNAK2 homology and acidic regions; } \\
\text { loss of interactions }\end{array}$ & [261] \\
\hline V882A & DSS & & {$[263,269]$} \\
\hline I921M & DSS & & [263] \\
\hline S929fs & DSS & $\begin{array}{l}\text { Loss of AHNAK2 homology and acidic regions; } \\
\text { loss of interactions }\end{array}$ & [263] \\
\hline K935E & DSS & & [263] \\
\hline K935stop & DSS & Loss of acidic domain; loss of integrin interaction & [263] \\
\hline $\mathrm{K} 1062 \mathrm{~N}$ & $\mathrm{CMT} 4 \mathrm{~F}$ & (Loss of predicted AnxA2/S100A10 interaction?) & [257] \\
\hline R1070stop & CMT4F & Loss of acidic domain; loss of integrin interaction & {$[208,259,267,270-272]$} \\
\hline P1083R & DSS & & [265] \\
\hline E1085fs & CMT4F & Loss of acidic domain; loss of integrin interaction & [273] \\
\hline K1095fs & CMT4F & Loss of acidic domain; loss of integrin interaction & [274] \\
\hline G1132R & DSS & & [263] \\
\hline E1259K & DSS & & [263] \\
\hline $\mathrm{R}_{1335 \mathrm{Q}^{2}}$ & CMT & & [266] \\
\hline E1359del & DSS & & [263] \\
\hline R1411C & DSS & & [263] \\
\hline
\end{tabular}

${ }^{1}$ fs denotes frame shift mutation, stop denotes nonsense mutation. ${ }^{2}$ Found together with V525A in a complex neuropathy associated with dysarthria, hypermobile joints, and cerebellar symptoms.

The authors apologize for any inconvenience caused. The original article has been updated.

\section{References}

1. Raasakka, A.; Kursula, P. Flexible Players within the Sheaths: The Intrinsically Disordered Proteins of Myelin in Health and Disease. Cells 2020, 9, 470. [CrossRef] [PubMed]

2. Yang, Y.; Shi, Y. Retraction: Spectrin-like domain 2 of DRP2 serves as a novel binding region for the NLS2 and 3 sub-domains of L-periaxin. RSC Adv. 2021, 11, 15160. [CrossRef]

3. Yang, Y.; Liang, M.; Shi, Y. Retraction: Self-association of L-periaxin occurs via its acidic domain and NLS2/NLS3, and affects its trafficking in RSC96 cells. RSC Adv. 2021, 11, 15203. [CrossRef] 